\title{
Emergency Noninvasive Angiography for Acute Intracerebral Hemorrhage
}

\author{
H. Khosravani, S.A. Mayer, A. Demchuk, B.S. Jahromi, D.J. Gladstone, M. Flaherty, J. Broderick, and R.I. Aviv
}

\begin{abstract}
SUMMARY: Spontaneous ICH is a devastating condition and is associated with significant mortality in the acute phase due to ongoing hemorrhage and hematoma expansion. A growing body of evidence suggests that there may be considerable utility in performing noninvasive vascular imaging during the acute-to-early phase of ICH. CTA has become widely available and is sensitive and specific for detecting vascular causes of secondary ICH such as aneurysms, arteriovenous malformations, dural arteriovenous fistulas, intracranial dissections, and neoplasm. CT venography can also diagnose dural sinus thrombosis presenting as hemorrhagic infarction. Recent data from stroke populations demonstrate a relatively low risk to patients when contrast is administered in the absence of a known serum creatinine. Detection of acute contrast extravasation within the hematoma ("spot sign") with CT angiography is predictive of subsequent hematoma expansion and is associated with increased morbidity and mortality. Risk stratification based on acute CTA can inform and expedite decision-making regarding intensive care unit admission, blood pressure control, correction of coagulopathy, and neurosurgical consultation. Noninvasive vascular imaging should be considered as an important component of the initial diagnostic work-up for patients presenting with acute $\mathrm{ICH}$.
\end{abstract}

ABBREVIATIONS: AHA/ASA = American Heart Association/American Stroke Association; $\mathrm{CAA}=$ cerebral amyloid angiopathy; $\mathrm{CIN}=$ contrast-induced nephropathy; $\mathrm{ICH}=$ intracerebral hemorrhage; $\mathrm{SICH}=$ spontaneous intracerebral hemorrhage

$I_{1}^{c}$ $\mathrm{CH}$ is the most deadly and disabling form of stroke. The 1 -month mortality is $30 \%-50 \%$, with half of these deaths occurring within the first 48 hours. ${ }^{1} \mathrm{SICH}$ is defined as hemorrhage in the absence of trauma. The etiology of hemorrhage is classified as either primary or secondary. Primary ICH usually occurs in older patients and is often associated with hypertension or CAA. Causes of secondary ICH include AVMs, aneurysms, anticoagulation, or hemorrhage due to neoplasm, infarction, or sinus thrombosis. ${ }^{2}$ Early imaging is elemental to diagnosing and triaging patients to appropriate care.

NCCT is the cornerstone of initial diagnostic evaluation in

From the Division of Neurology (H.K., D.J.G.) and Department of Medical Imaging (R.I.A.), Department of Medicine, Sunnybrook Health Science Centre, University of Toronto, Toronto, Ontario, Canada; Department of Neurology (S.A.M.), The Neurological Institute of New York, Columbia University Medical Center, New York, New York; Department of Clinical Neurosciences and Radiology (A.D.), Calgary Stroke Program, Hotchkiss Brain Institute, University of Calgary, Calgary, Alberta, Canada; Departments of Neurosurgery, Neurology, and Imaging Sciences (B.S.J.), University of Rochester Medical Center, University of Rochester, Rochester, Minnesota; and Department of Neurology (M.F., J.B.), University of Cincinnati Health University Hospital, University of Cincinnati, Cincinnati, Ohio.

Please address correspondence to Richard I. Aviv, MD, University of Toronto and Sunnybrook Health Science Centre, Room AG 31, 2075 Bayview Ave, Toronto, Ontario M4N 3M5, Canada; e-mail: richard.aviv@sunnybrook.ca

- Indicates open access to non-subscribers at www.ajnr.org

三 Indicates article with supplemental on-line table.

http://dx.doi.org/10.3174/ajnr.A3296 patients with ICH, given its high sensitivity for acute hemorrhage and wide availability. DSA remains the criterion standard imaging technique for characterization and detection of secondary lesions. ${ }^{1,3,4}$ However, DSA is invasive with possible significant deleterious effects to the patient, in addition to being costly and not readily available. This renders DSA suboptimal as a screening test. Findings from early studies with small series of patients with ICH suggested that hypertension together with certain ICH locations (basal ganglia, thalamus, cerebellum, and pons) could reliably exclude underlying secondary lesions. ${ }^{5}$ These findings led to a widely held view that searching for secondary lesions should be reserved for younger individuals with nontypical ICH locations without hypertension or those with IVH.

Studies using CTA have demonstrated, conservatively, a $2 \%-4 \%$ prevalence of secondary vascular lesions even in patients with typical hypertensive hemorrhage locations previously excluded from DSA. ${ }^{6-8}$ CTA is widely available and has a proved track record in subarachnoid hemorrhage ${ }^{9}$ and is increasingly part of standard imaging protocols in acute stroke ${ }^{10}$ and trauma. The effectiveness of CTA in detecting lesions, its availability, and patient tolerability render it a useful noninvasive screening technique. Recent imaging advances facilitate detection of secondary lesions in $\mathrm{SICH}$ with good accuracy ${ }^{7,11}$ and patient safety. ${ }^{12-14}$ Furthermore, the prevalence of secondary vascular lesions is sim- 
ilar to that of occult C-spine fractures in the acute trauma setting, where extended emergent CT is established, showing favorable safety and cost-effectiveness profiles. ${ }^{15,16}$

Early neurologic deterioration frequently results from hematoma expansion, occurring in 30\% of patients with ICH within 3 hours of symptom onset. ${ }^{17-19}$ Secondary lesions also have a higher rebleed rate, impacting patient outcomes. The risk of early rebleed in aneurysmal SAH is $\leq 12 \%$, with significant morbidity and mortality. ${ }^{20}$ Rehemorrhage risk from AVM is $7 \%$ in the first year, thereafter reducing to $2 \%-4 \%$ annual risk. ${ }^{21}$ Recently, the presence of contrast extravasation ("spot sign") on CTA has been associated with increased risk of hematoma expansion and poor outcome in both retrospective and prospective studies. ${ }^{22-28}$ Identification of vascular lesions and patients at risk for early hematoma expansion may improve outcome by allowing directed clinical decision-making. ${ }^{29}$

We review the current diagnostic guidelines for $\mathrm{ICH}$ and discuss imaging considerations. We provide an evidence-based framework for the superiority of noninvasive angiography as a screening tool. Recent developments relating to angiographic markers associated with hematoma expansion are reviewed, and developments surrounding the safety profile of noninvasive vascular imaging are highlighted. We focus on CTA as the prototype vascular imaging in SICH, given that it is widely available, used, and studied within acute SICH. We propose a pathway for radiologic evaluation of patients and propose that CTA should be considered as part of routine imaging in the initial diagnostic work-up of patients with acute SICH.

\section{CURRENT GUIDELINES FOR IMAGING PATIENTS WITH ICH}

It is not possible to definitively differentiate ICH from ischemic stroke or SAH without imaging, but $\mathrm{ICH}$ often presents with a typical clinical syndrome. Suggestive features include sudden onset of a progressive neurologic deficit, severe hypertension, and impaired level of consciousness. Emesis and headache are also frequently present.

The AHA/ASA guidelines indicate that either NCCT or MR imaging may be used in the initial diagnostic evaluation of ICH. ${ }^{1,3,4} \mathrm{~T}^{*}$ susceptibility-weighted MR imaging is considered equivalent to NCCT for identifying acute blood. ${ }^{4}$ Additionally, MR imaging may provide information about hemorrhage timing, degree of perihematomal edema, infarction, and the presence of microbleeds or cavernoma. ${ }^{4}$ In most centers, however, NCCT remains the technique of choice due to its availability, patient tolerability, reduced cost, and scan duration. Extrapolating from acute stroke imaging studies, we found that MR imaging is limited by patient contraindications and/or patient tolerability factors, including depressed level of consciousness, airway control, hemodynamic instability, agitation, or metallic implants. ${ }^{30}$

The 1999/2007 AHA/ASA guidelines recommended angiography (DSA or CTA) for all patients without a clear cause for hemorrhage, who are surgical candidates, and particularly in young and normotensive patients who are clinically stable. ${ }^{1}$ Other emphasized indications for angiography include the presence of subarachnoid hemorrhage and abnormal calcifications and patients with isolated IVH. ${ }^{3}$ The guidelines indicated that DSA is not re- quired in older hypertensive patients with so-called typical hypertensive bleeds within the basal ganglia, thalamus, cerebellum, or brain stem unless the NCCT findings are suspicious for an underlying lesion. This statement is based on the low diagnostic yield of angiography in this subset weighed against the risks of conventional angiography. Use of DSA requires consideration of patient factors and a balance between the need for diagnostic information and potential timing of surgical intervention (such as during symptoms of herniation).

The most recent AHA/ASA guidelines (2010) acknowledge a wider and more flexible role for noninvasive vascular imaging in $\mathrm{ICH}$, including CT/MR imaging angiography/venography. ${ }^{4}$ Specifically, the decision to obtain vascular imaging is deferred to the care team without an emphasis on the patient's clinical risk factors or the identification of NCCT features that may be considered suspicious for underlying hemorrhage.

\section{CONSIDERATIONS FOR PERFORMING ROUTINE CT ANGIOGRAPHY IN PATIENTS WITH ICH Limitations of NCCT as a Solitary Diagnostic Technique}

NCCT is a widely used screening technique in distinguishing ischemic from hemorrhagic acute stroke. There is a notion that hemorrhage location (eg, deep structures, lobar) and associated imaging attributes may infer a particular underlying primary etiology. However, this approach is poorly sensitive and may miss secondary causes in patients with pre-existing hypertension. ${ }^{7,8,31}$

Few studies have systematically examined the performance of NCCT in detecting secondary lesions. In 1 prospective study, patients with ICH were classified according to the presence or absence of suspicious features on NCCT. However, NCCT alone demonstrated a poor sensitivity (77\%) and specificity (84\%) relative to DSA. ${ }^{31}$ In a retrospective study, Delgado Almandoz et al ${ }^{6}$ categorized 623 patients with ICH into low, intermediate, and high probability of vascular lesions on the basis of NCCT characteristics. More than two-thirds of patients could not be confidently classified with NCCT and were assigned to an intermediate-risk category. Only 3\% demonstrated suspicious NCCT features for an underlying lesion. In a follow-up study, ${ }^{32}$ the sensitivity and specificity for secondary lesions were $20 \%$ and $99 \%$, respectively, if intermediate NCCT appearances were considered together with low-risk patients. If intermediate and high risk groups were considered together, the performance was $95 \%$ and $35 \%$, respectively. A SICH score was recently published using clinical features and NCCT to stratify the risk for underlying lesions. ${ }^{32}$ A positive correlation was shown between a higher score and secondary lesions. ${ }^{33}$ However, low prevalence of suspicious features on NCCT remains a significant limitation of such scores.

\section{SECONDARY VASCULAR LESIONS IN PATIENTS WITH SICH ARE COMMON}

A number of studies have evaluated the incidence of secondary vascular causes in patients with SICH. Overall, the incidence of vascular etiologies in ICH as detected by pathology and/or angiography ranges between $14 \%$ and 53\% (On-line Table and Table 1)..$^{5,6,8,31,32,34,35}$ All studies are limited by small sample sizes and verification or selection bias.

Early small series reinforced a currently entrenched dogma 


\begin{tabular}{|c|c|c|c|c|c|c|c|}
\hline Study & AVM & Aneurysm & $\begin{array}{c}\text { Venous } \\
\text { Sinus } \\
\text { Thrombosis }\end{array}$ & Moyamoya $^{a}$ & $\begin{array}{l}\text { Arterial or } \\
\text { Venous } \\
\text { Malformation }\end{array}$ & $\begin{array}{l}\text { Mass } \\
\text { Lesion }\end{array}$ & $\begin{array}{c}\text { Negative } \\
\text { Angiography } \\
\text { Findings }\end{array}$ \\
\hline Toffol et al, $1986^{5}$ & $9 \%$ & $12 \%$ & $3 \%$ & $3 \%$ & - & - & $72 \%$ \\
\hline Halpin et al, $1994^{31}$ & $39 \%$ & $14 \%$ & - & & - & - & $47 \%$ \\
\hline Zhu et al, $1997^{8}$ & $25 \%$ & $5 \%$ & $2 \%$ & $2 \%$ & - & - & $66 \%$ \\
\hline Griffiths et al, $1997^{34}$ & $27 \%$ & $22 \%$ & $1 \%$ & - & - & - & $50 \%$ \\
\hline Hino et al, $1998^{40}$ & $24 \%$ & $2 \%$ & - & - & $4 \%$ & $3 \%$ & $70 \%$ \\
\hline Yeung et al, $2009^{7}$ & $24 \%$ & $13 \%$ & - & $2 \%$ & $7 \%$ & $5 \%$ & $49 \%$ \\
\hline Delgado Almandoz et al, $2009^{6}$ & $7 \%$ & $4 \%$ & $3 \%$ & $0.3 \%$ & $2 \%$ & & $84 \%$ \\
\hline Delgado Almandoz et al, $2010^{32 b}$ & $9 \%$ & $3 \%$ & $2 \%$ & $1 \%$ & $2 \%$ & - & $84 \%$ \\
\hline
\end{tabular}

Note: - - indicates data not available.

${ }^{a}$ Moyamoya refers to congenital Moyamoya disease and/or acquired Moyamoya-like syndrome secondary to chronic intracranial arterial steno-occlusive disease.

b Prospective cohort only who had multidetector CTA imaging.

that hemorrhage in the basal ganglia is almost always of hypertensive etiology. ${ }^{36-38}$ However, elderly patients are often considered unlikely to harbor secondary vascular causes and may not be investigated. ${ }^{8}$ An early retrospective SICH study demonstrated that DSA detected more lesions in nonhypertensive (22/50) compared with hypertensive patients $(6 / 47) .{ }^{5}$ In a prospective study evaluating the application of angiography in $\mathrm{ICH}$, Halpin et $\mathrm{al}^{31}$ performed DSA following initial NCCT for a subset of patients with suspicious NCCT features suggesting a structural lesion. Underlying vascular lesions were seen in 42/82 (51\%) of all patients and in $13 \%$ of hypertensive patients. Locations traditionally associated with hypertensive hemorrhage, such as the basal ganglia and posterior fossa, demonstrated vascular lesions in 31\% and $18 \%$, respectively. A subset of patients with $\mathrm{ICH}$ without suspicious features on NCCT underwent delayed angiography, revealing a lesion in $24 \%$ of patients. Patient selection factors likely biased their results toward higher frequency of structural lesions. However, the incidence of underlying vascular lesions in hypertensive patients was similar to that in previous studies. ${ }^{5,39,40}$

A pathologic series of 144 brains demonstrated a secondary cause for ICH in $36 \%$ of hypertensive patients. ${ }^{41}$ Thirty percent were due to AVMs and aneurysms. The high lesion frequency may have been confounded by patient referral/selection bias - typical cases of ICH in the setting of hypertension and older patients are much less likely to have a postmortem examination. In a prospective study of 100 patients with ICH, 58\% of normotensive patients and $25 \%$ of hypertensive patients had abnormalities on angiography. ${ }^{34}$ These authors acknowledged the lower diagnostic yield in hypertensive patients but advocated for CTA, arguing that AVMs and hypertension can coexist in older patients without a clear relation to clinical and NCCT findings. A prospective study of lobar ICH in 78 patients compared the diagnostic yield of CTA with DSA, showing secondary lesions in $28 \%$ of patients and diagnostic equivalence of CTA to DSA. ${ }^{11}$

Zhu et $\mathrm{al}^{8}$ examined a population of 206 patients with DSA. DSA detected lesions in $34 \%$ of patients, and findings were positive in $50 \%$ of those younger than 45 years compared with $18 \%$ for those older than 45 years. A more recent study similarly showed a DSA yield of 39\% in older patients with ICH compared with 55\% in patients younger than 45 years. ${ }^{7}$ Zhu et $\mathrm{al}^{8}$ also showed that DSA findings were positive in $9 \%$ of patients with underlying hypertension compared with $45 \%$ of nonhypertensives. However, there were 2.5 times more nonhypertensives than hypertensives in their study. Therefore, it is plausible that the inclusion of fewer hypertensive patients underestimated the incidence of secondary lesions, ${ }^{31}$ and the incidence of secondary lesions in younger hypertensive patients was undetermined due to small sample size.

Delgado Almandoz et $\mathrm{al}^{6}$ retrospectively studied 623 patients with ICH who underwent NCCT and CTA. Overall, 15\% (91/623) of patients had a detectable vascular lesion on CTA. Notably, $11 \%$ (70/623) were older than 45 years of age and had pre-existing hypertension. The incidence was lower $(\sim 4 \%)$ for patients older than 71 years of age (11/273). Overall, the highest CTA yield occurred in female patients younger than 45 years with lobar or infratentorial hemorrhage, IVH, and lack of known hypertension or coagulopathy-34\% (210/623) of patients had CTA and DSA or direct surgical and pathologic correlation.

\section{ICH LOCATION AND CLINICAL CRITERIA ALONE CANNOT RULE OUT A VASCULAR LESION}

Approximately one-half of ICH cases originate in the basal ganglia; a third, in the cerebral hemispheres; and a sixth, in the brain stem or cerebellum. ${ }^{2}$ In $\leq 40 \%$ of the cases, there is accompanying intraventricular hemorrhage. ${ }^{42}$ Halpin et al ${ }^{31}$ described 26 lesions occurring in the basal ganglia with a secondary cause in $31 \%$ (AVM in 23\% and aneurysm in $8 \%$ ). Basal ganglia location was defined as any combination of caudate, globus pallidus, putamen, thalamus, internal/external capsule, and insula. In the study of Zhu et al, ${ }^{8} 49 \%$ of patients with a lobar hemorrhage had positive findings on DSA; $19 \%$ of these patients had hypertension. Older normotensive patients with basal ganglia bleeds had a 7\% incidence of underlying vascular lesions. Of the patients with positive DSA findings, 13\% had basal ganglia hemorrhages when including the thalamus and caudate. Overall, the incidence of a basal ganglia lesion, in both this and a similar study, was $4 \% .^{7,8}$ In a retrospective review comparing CTA and DSA for secondary ICH etiology, an underlying vascular lesion was present in $2.6 \%$ of patients with a basal ganglia bleed location. ${ }^{6}$ In summary, the incidence of a vascular lesion in a typical basal ganglia hypertensive location is minimally $4 \%$ (Table 2 ).

\section{SPOT SIGN PREDICTS EARLY ICH GROWTH}

Foci of arterial phase contrast enhancement, coined the "spot sign" within acute ICH, are easily identified with high interobserver agreement. ${ }^{27}$ CTA spot sign prevalence is $20 \%-30 \%$ within 
Table 2: Prevalence of secondary lesions in the basal ganglia ${ }^{a}$ relative to the total number of lesions identified by DSA and listed by etiology

No Secondary

Lesion Lesion

Study AVM Aneurysm Other Identified Identified

Halpin et al, $1994^{31} 4 \%(6) \quad 1 \%(2) \quad 7 \%(10) \quad 5 \%(8) \quad 12 \%$

Zhu et al, $1997^{8} \quad 2 \%(5) \quad 1 \%(2) \quad 1 \%(2) \quad 34 \%(70) \quad 4 \%$

$\begin{array}{llllll}\text { Yeung et al, } 2009^{7} & (0) & 4 \%(2) & (0) & 13 \%(7) & 4 \%\end{array}$

Note:-Numbers in parentheses indicate the number of patients.

a Basal ganglia location is defined as any combination of caudate, globus pallidus, putamen, and thalamus.

3-10 hours of ictus. Enhancement is thought to represent extraluminal extravasation and may appear serpiginous to spotlike, single, or multiple; occur within the hematoma without identifiable external vessel connection; and demonstrate an attenuation approximately double that of the surrounding hematoma. ${ }^{43}$

Data from both retrospective and prospective studies indicate that the CTA spot sign is a marker of hemorrhage expansion, ${ }^{24,26,27,44}$ though timing of CTA impacts the likelihood of growth determination. ${ }^{45-48}$ The spot sign is highly predictive of hematoma expansion with a positive likelihood ratio of $\sim 8.5$. The spot sign has a positive predictive value of $\sim 75 \%$ and a negative predictive value in the range of $85 \%-95 \% .^{24,26-28}$ The variability of positive and negative predictive values may arise due to differences of CTA timing and whether delayed image acquisitions were used. ${ }^{28}$ CTA contrast extravasation also independently predicts in-hospital mortality. ${ }^{22,25,26}$ A prospective study demonstrated that contrast extravasation on CTA is a significant and independent predictor of poor clinical outcome with an odds ratio of $>10 .{ }^{26}$

Recent results from a multicenter prospective spot sign validation study, Prediction of Haematoma Growth and Outcome in Patients with Intracerebral Haemorrhage Using the CT-Angiography Spot Sign, confirmed earlier single-center observations, though it showed a lower sensitivity and positive predictive value than previously reported ( $50 \%$ and $58 \%$, respectively). Specificity $(85 \%)$ and negative predictive value $(80 \%)$ remained robust. $^{28,45,49}$ The spot sign has been detected with similar frequency in basal ganglia and lobar hemorrhages. ${ }^{50}$ A spot sign score has been devised that stratifies the risk of hematoma expansion and clinical outcome. ${ }^{48}$ However, further evaluation for each score level showed that the optimal performance for hematoma expansion occurs in the presence of a spot sign, independent of its size or contrast attenuation. The clinical utility of the spot sign score has therefore been questioned. ${ }^{47,48}$

The spot sign has not been observed in secondary ICH; this finding suggests some specificity for spontaneous $\mathrm{ICH} .{ }^{51}$ The identification of this radiographic sign has important implications for predicting the risk of hematoma expansion and prognosis and potentially as a surrogate marker for considering hemostatic therapy. An important addition to the 2010 AHA/ASA guidelines is the recommendation that spot sign detection may stratify patients at a higher risk for hematoma expansion. ${ }^{4}$

\section{CT ANGIOGRAPHY ACCURACY APPROACHES THAT OF DSA}

DSA is the reference standard for diagnosing vascular lesions but is invasive and associated with $0.9 \%$ and $0.5 \%$ risk of transient and permanent neurologic deficits, respectively. DSA is also expensive and resource-intensive. These factors limit routine use of DSA in ICH. ${ }^{52}$ Historical DSA data therefore have selection bias. Relatively, noninvasive vascular imaging is associated with a high safety profile and low complication rate and may be applied to patients who may otherwise have been excluded from DSA. DSA remains appropriate in the context of CTA-detected vascular lesions and where ongoing high clinical suspicion remains in the presence of normal noninvasive test findings. Similar algorithms are established for SAH. ${ }^{9,53}$ DSA is time- and labor-intensive, requiring patient cooperation, clinical stability, and monitoring. Radiation dose is delivered to both patient and operator, and contrast doses may exceed those of CTA. Recent studies comparing CTA with DSA suggest the high accuracy of CTA for diagnosis of underlying vascular anomalies, with sensitivities of $\geq 95 \%$ and specificity approaching $100 \%$. Positive and negative predictive values are in excess of $97 \% .{ }^{11,54}$ However, CTA imaging remains inferior to DSA for temporal imaging of vessels; this feature limits its ability to elucidate important feeding branches and drainage pathways of vascular malformations. ${ }^{6,54}$ Newer multidetector row CT scanners have improved temporal resolution but are associated with significant increases in radiation dose. Finally, the spatial resolution of multidetector row CT remains inferior to that of DSA. ${ }^{55}$ Overall, CTA is an effective initial screen for identifying secondary vascular lesions and may replace DSA in certain clinical situations (Fig 1).

\section{EMERGENT NONINVASIVE VASCULAR IMAGING DOES NOT REQUIRE A SERUM CREATININE LEVEL IN APPROPRIATE PATIENTS}

The potential for deleterious effects on renal function applies equally to CTA and DSA. CIN is defined as a $25 \%$ elevation from baseline serum creatinine levels or an absolute increase of 0.5 $\mathrm{mg} / \mathrm{dL}$ within $48-72$ hours of contrast administration. ${ }^{56}$ Chronic renal impairment is the most important risk factor for the development of CIN. The absence of risk factors effectively eliminates the probability of renal impairment in most patients. ${ }^{57}$ An elevated baseline creatinine level, though indicative of pre-existing nephropathy, does not reliably predict CIN development. ${ }^{12,56}$ The estimated incidence of $\mathrm{CIN}$ is $2 \%-7 \%$. A retrospective review of patients undergoing CTA reported a $2 \%$ incidence of CIN in patients given a low-osmolar contrast agent and 3\% for the entire cohort $^{58} ; 9 \%$ of patients were on metformin and $0.9 \%$ and $38 \%$ had known renal impairment and diabetes, respectively. No patients went on to require dialysis. In a prospective study of 575 patients with stroke investigated with CTA, CTA/CTP, and CTA/ CTP/DSA, the risk of CIN was 5\%. ${ }^{14}$ After adjustment for confounding factors, the incidence of CIN in a similar non-contrastexposed stroke group was $10 \%$. Comparison with historical controls before routine CTA use has confirmed a higher incidence of renal impairment in patients with stroke not receiving contrast. ${ }^{59}$ The authors suggest that CIN be renamed "hospital-induced nephropathy," reflecting homeostatic alterations in the hospitalized patient. ${ }^{60,61}$ This notion was recently alluded to in a study involving patients with $\mathrm{ICH}$, in which CTA was not associated with an increased risk of CIN. ${ }^{13}$

Recent guidelines recommend that when possible, patients 


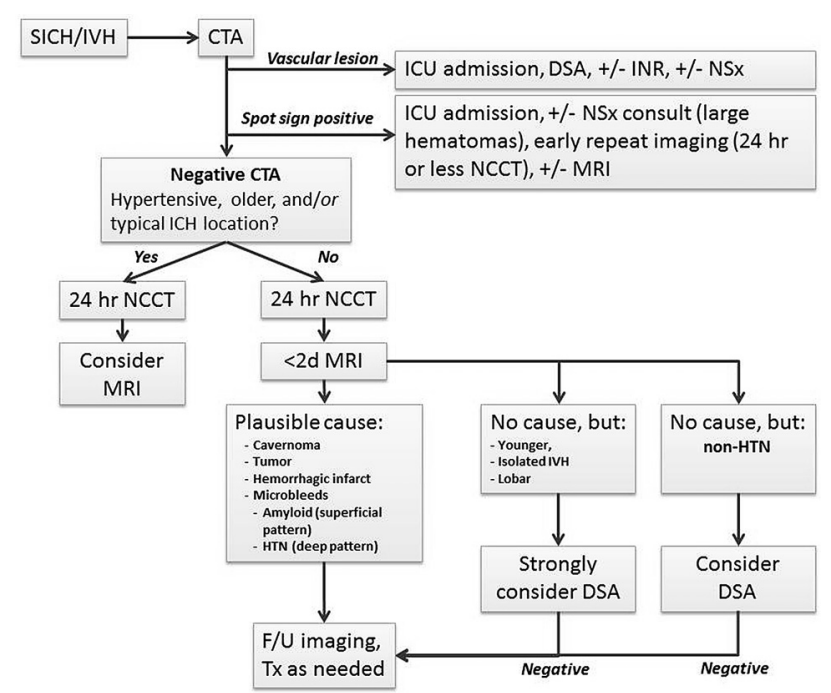

FIG 1. A suggested protocol for SICH investigation on acute presentation to a hospital.

should be screened for risk factors associated with acute or chronic renal impairment. ${ }^{62}$ In the presence of risk factors, empiric precautions of hydration and iso-osmolar contrast agent use are recommended. Patients with a normal glomerular filtration rates are at extremely low risk of $\mathrm{CIN}$ and require no specific prophylaxis or follow-up. Risk-factor exclusion may be impossible in the acute setting, and the delay in obtaining laboratory investigations should be balanced against the potential negative impact on patient outcome. ${ }^{62}$ Notably, CTA-based stroke and body trauma protocols are often implemented without knowledge of contrast allergy or renal function. ${ }^{58,63-66}$ Overall, there is a very low incidence of adverse events and mortality. ${ }^{67}$ These diagnostic imaging considerations, as they relate to the patient's clinical status and safety, are acknowledged in the AHA/ASA ICH guidelines.

\section{THE RISK-TO-BENEFIT RATIO OF INCREMENTAL RADIATION EXPOSURE WITH CTA IS REASONABLE}

Radiation dose remains a concern for CT-based noninvasive imaging. In a study by Cohnen et al, ${ }^{68}$ the intracranial CTA dose was not markedly different from that in NCCT (1.9 mSV compared with $1.7 \mathrm{mSV}$, respectively). CTA performed from the aortic arch to the vertex is associated with a higher dose of 2.8-3.4 mSV due to the increased coverage. The estimated lifetime risk of death from cancer that is attributable to a single head CT scan is welldocumented at an estimated 0.01\%-0.02\%. ${ }^{69}$ Repeated lifetime scanning is associated with proportional increases in cancer risk. ${ }^{70}$ Therefore, clinical judgment still needs to be exercised before vascular imaging, despite the devastating nature of $\mathrm{ICH}$.

\section{PROPOSED DIAGNOSTIC IMAGING ALGORITHM FOR ACUTE ICH}

Appropriate application of diagnostic imaging modalities can have a meaningful impact on initial management (medical or invasive) and may inform care providers of the risk of early clinical deterioration. An effective imaging strategy in patients with ICH is one that is readily available, accessible, rapid, well-tolerated, and accurate for lesion detection.
Given that vascular imaging has some degree of risk, albeit small, a balanced viewpoint may be the best approach for individual patients. We have outlined the case for using noninvasive vascular imaging to search for lesions in patients previously thought least likely to have them: older hypertensive patients. It is conceivable that some patients within this grouping, with NCCT ICH in a typical location and without any suspicious features, may indeed have a secondary lesion but one that is likely not treatable (eg, due to patient comorbidities). When this possibility is balanced against potential risks of vascular imaging, it may be appropriate in some patients to forego additional imaging. As an extension, it may be argued that CTA could be delayed until a creatinine level is available because of concerns for CIN, despite a large and convincing body of evidence against this. In contrast, detection of a secondary lesion and the presence of a spot sign, indicating the likelihood of hematoma growth and the potential for clinical deterioration, not only impact management and prognosis but also the discussion a clinician has with a patient's relatives in the acute setting.

Emerging data regarding the prevalence of secondary lesions and a marker of hematoma expansion argue for early CTA. It has been shown that in related emergency situations, complete initial CT imaging protocols are more cost-effective. We recommend CTA acutely at the time of initial NCCT in most patients with appropriate considerations. A suggested algorithm for the investigation of patients with ICH based on a synthesis of the reviewed literature and existing AHA/ASA guidelines is presented (Fig 1).

In the presence of a vascular lesion, patients are diverted to DSA and possible interventional (interventional neuroradiology or neurosurgical) management. Early neurosurgical consultation may be warranted in spot sign-positive patients with large hematomas. In the absence of CTA findings, patients are then dichotomized to hypertensive (older patients with typical bleed locations) or normotensive groups according to current practice. In select patients, perhaps those with lobar ICH, it may be reasonable to proceed with MR imaging to look for microbleeds (eg, CAA) or other etiologies (neoplasm) that impact decision-making regarding antithrombotic agents. In patients with positive findings on CTA, physicians follow the established decision pathway of the AHA/ASA guidelines. ${ }^{1,3,4}$ Both groups receive 24-hour follow-up CT to assess hematoma growth (or sooner, if clinically deteriorating). We advocate early MR imaging/MRA (with or without gadolinium according to local practice) to look for microbleeds or angiographically occult lesions such as cavernoma or neoplasm. CTA-negative normotensive patients younger than 45 years, patients with isolated IVH, and those with lobar hemorrhage in the absence of microbleeds on MR imaging should be strongly considered for a DSA. However, clinical judgment needs to be exercised as to whether such diagnostic information is going to be beneficial for all patients, given that a patient's management may not be impacted by this additional information. CTA-negative normotensive patients older than 45 years may occasionally be considered for DSA at the discretion of the treating physician. ${ }^{4,8}$ Follow-up imaging, in the form of a remote MR imaging at 6-8 weeks (or later), is useful to document the evolution of the ICH and to exclude an abnormality masked by the hematoma. 


\section{FUTURE DIRECTIONS}

There are no clinical or NCCT characteristics that reliably predict ICH etiology. The advent of a potential marker of hematoma growth argues for a paradigm shift in ICH imaging. Limitations of safety and resource availability that hampered DSA no longer apply in the context of highly sensitive and low-risk noninvasive vascular imaging. Overall, the risk of a secondary vascular cause in ICH is approximately $30 \% .^{5,6,8,31,32,34,35}$ Hypertensive patients, traditionally excluded from vascular imaging due to typical locations of hemorrhages, may harbor a lesion in approximately $4 \%$ of cases. Noninvasive vascular imaging has a role in detecting structural lesions but also identifies patients at risk of hematoma expansion.

While the impact of hemostatic therapies on the spot sign is currently unknown, CTA has identified a potent and reliable marker of hematoma expansion and poor outcome, which is seen in $\sim 20 \%-30 \%$ of all patients with ICH. The ongoing studies Spot Sign for Predicting and Treating ICH Growth and Spot Sign Selection of Intracerebral Hemorrhage to Guide Hemostatic Therapy will provide prospective validation of the spot sign as a predictor of hematoma expansion and secondarily investigate the biologic and clinical effect of recombinant activated factor VIIa treatment in the setting of the CTA spot sign.

Disclosures: Andrew Demchuk_UNRELATED: Grants/Grants Pending: Canadian Institute of Health Research Spot Sign Selection of Intracerebral Hemorrhage to Guide Hemostatic Therapy (SPOTLIGHT) trial, ${ }^{*}$ NIH Spot Sign for Predicting and Treating ICH Growth (STOP-IT) trial CT core laboratory, ${ }^{*}$ Other: member of executive committee, ${ }^{*}$ SPOTLIGHT trial.* David Gladstone-UNRELATED: Other: Dr Gladstone is the Principal Investigator of the Canadian Institute of Health Research-funded SPOTLIGHT trial investigating recombinant factor Vlla for patients with CTA spot sign-positive intracerebral hemorrhages. Matthew Flaherty-UNRELATED: Consultancy: Boerhinger Ingelheim, Grants/Grants Pending: National Institute of Neurological Disorders and Stroke, Patents (planned, pending, or issued): Dr Flaherty is coinventor of a noninvasive CNS-monitoring device for which a patent is pending, Other: advisory board, CSL Behring. Joseph Broderick—RELATED: Grant: Novo Nordisk, ${ }^{*}$ Comments: supplies drug for National Institute of Neurological Disorders and Stroke-funded STOP-IT trial. I am the Principal Investigator of the overall National Institute of Neurological Disorders and Stroke Specialized Programs of Translational Research in Acute Stroke study, which includes STOP-IT. Richard Aviv-UNRELATED: Grants/Grants Pending: Canadian Institute of Health Research. * Money paid to the institution.

\section{REFERENCES}

1. Broderick JP, Adams HP Jr, Barsan W, et al. Guidelines for the management of spontaneous intracerebral hemorrhage: a statement for healthcare professionals from a special writing group of the Stroke Council, American Heart Association. Stroke 1999;30:905-15

2. Mayer SA, Rincon F. Treatment of intracerebral haemorrhage. Lancet Neurol 2005;4:662-72

3. Broderick J, Connolly S, Feldmann E, et al. Guidelines for the management of spontaneous intracerebral hemorrhage in adults: 2007 update: a guideline from the American Heart Association/American Stroke Association Stroke Council, High Blood Pressure Research Council, and the Quality of Care and Outcomes in Research Interdisciplinary Working Group. Stroke 2007;38:2001-23

4. Morgenstern LB, Hemphill JC 3rd, Anderson C, et al. Guidelines for the management of spontaneous intracerebral hemorrhage: a guideline for healthcare professionals from the American Heart Association/American Stroke Association. Stroke 2010;41:2108-29

5. Toffol GJ, Biller J, Adams H Jr, et al. The predicted value of arteriography in nontraumatic intracerebral hemorrhage. Stroke 1986;17: 881-83

6. Delgado Almandoz JE, Schaefer PW, Forero NP, et al. Diagnostic accuracy and yield of multidetector CT angiography in the evalua- tion of spontaneous intraparenchymal cerebral hemorrhage. AJNR Am J Neuroradiol 2009;30:1213-21

7. Yeung R, Ahmad T, Aviv RI, et al. Comparison of CTA to DSA in determining the etiology of spontaneous ICH. Can J Neurol Sci 2009;36:176-80

8. Zhu XL, Chan MS, Poon WS. Spontaneous intracranial hemorrhage: which patients need diagnostic cerebral angiography? A prospective study of 206 cases and review of the literature. Stroke 1997;28:1406-09

9. Agid R, Lee SK, Willinsky RA, et al. Acute subarachnoid hemorrhage: using 64-slice multidetector CT angiography to "triage" patients' treatment. Neuroradiology 2006;48:787-94

10. Smith WS, Roberts HC, Chuang NA, et al. Safety and feasibility of a CT protocol for acute stroke: combined CT, CT angiography, and CT perfusion imaging in 53 consecutive patients. AJNR Am J Neuroradiol 2003;24:688-90

11. Yoon DY, Chang SK, Choi CS, et al. Multidetector row CT angiography in spontaneous lobar intracerebral hemorrhage: a prospective comparison with conventional angiography. AJNR Am J Neuroradiol 2009;30:962-67

12. Tippins RB, Torres WE, Baumgartner BR, et al. Are screening serum creatinine levels necessary prior to outpatient CT examinations? Radiology 2000;216:481-84

13. Oleinik A, Romero JM, Schwab K, et al. CT angiography for intracerebral hemorrhage does not increase risk of acute nephropathy. Stroke 2009;40:2393-97

14. Lima FO, Lev MH, Levy RA, et al. Functional contrast-enhanced CT for evaluation of acute ischemic stroke does not increase the risk of contrast-induced nephropathy. AJNR Am J Neuroradiol 2010;31: 817-21

15. Blackmore CC, Ramsey SD, Mann FA, et al. Cervical spine screening with CT in trauma patients: a cost-effectiveness analysis. Radiology 1999;212:117-25

16. Hanson JA, Blackmore CC, Mann FA, et al. Cervical spine injury: a clinical decision rule to identify high-risk patients for helical CT screening. AJR Am J Roentgenol 2000;174:713-17

17. Brott T, Broderick J, Kothari R, et al. Early hemorrhage growth in patients with intracerebral hemorrhage. Stroke 1997;28:1-5

18. Fujii $Y$, Tanaka R, Takeuchi S, et al. Hematoma enlargement in spontaneous intracerebral hemorrhage. J Neurosurg 1994;80:51-57

19. Kazui S, Naritomi H, Yamamoto H, et al. Enlargement of spontaneous intracerebral hemorrhage: incidence and time course. Stroke 1996;27:1783-87

20. Starke RM, Connolly ES Jr, for the Participants in the International Multi-Disciplinary Consensus Conference on the Critical Care Management of Subarachnoid Hemorrhage. Rebleeding after aneurysmal subarachnoid hemorrhage. Neurocrit Care 2011;15:241-46

21. Mattle HP, Schroth G, Seiler RW. Dilemmas in the management of patients with arteriovenous malformations. J Neurol 2000;247: 917-28

22. Becker KJ, Baxter AB, Bybee HM, et al. Extravasation of radiographic contrast is an independent predictor of death in primary intracerebral hemorrhage. Stroke 1999;30:2025-32

23. Ederies A, Demchuk A, Chia T, et al. Postcontrast CT extravasation is associated with hematoma expansion in CTA spot negative patients. Stroke 2009;40:1672-76

24. Goldstein JN, Fazen LE, Snider R, et al. Contrast extravasation on CT angiography predicts hematoma expansion in intracerebral hemorrhage. Neurology 2007;68:889-94

25. Kim J, Smith A, Hemphill JC 3rd, et al. Contrast extravasation on CT predicts mortality in primary intracerebral hemorrhage. AJNR Am J Neuroradiol 2008;29:520-25

26. Li N, Wang Y, Wang W, et al. Contrast extravasation on computed tomography angiography predicts clinical outcome in primary intracerebral hemorrhage: a prospective study of 139 cases. Stroke 2011;42:3441-46

27. Wada R, Aviv RI, Fox AJ, et al. CT angiography "spot sign" predicts hematoma expansion in acute intracerebral hemorrhage. Stroke 2007;38:1257-62 
28. Demchuk AM, Dowlatshahi D, Rodriguez-Luna D, et al. Prediction of haematoma growth and outcome in patients with intracerebral haemorrhage using the CT-angiography spot sign (PREDICT): a prospective observational study. Lancet Neurol 2012;11:307-14

29. van Gijn J, Rinkel GJ. Subarachnoid haemorrhage: diagnosis, causes and management. Brain 2001;124:249-78

30. Briley DP, Meagher T, King D. Practical limitations of acute stroke MRI due to patient-related problems. Neurology 2005;64:400-01, author reply 400-01

31. Halpin SF, Britton JA, Byrne JV, et al. Prospective evaluation of cerebral angiography and computed tomography in cerebral haematoma. J Neurol Neurosurg Psychiatry 1994;57:1180-86

32. Delgado Almandoz JE, Schaefer PW, Goldstein JN, et al. Practical scoring system for the identification of patients with intracerebral hemorrhage at highest risk of harboring an underlying vascular etiology: the Secondary Intracerebral Hemorrhage Score. AJNR Am J Neuroradiol 2010;31:1653-60

33. Delgado Almandoz JE, Jagadeesan BD, Moran CJ, et al. Independent validation of the secondary intracerebral hemorrhage score with catheter angiography and findings of emergent hematoma evacuation. Neurosurgery 2012;70:131-40, discussion 140

34. Griffiths PD, Beveridge CJ, Gholkar A. Angiography in non-traumatic brain haematoma: an analysis of 100 cases. Acta Radiol 1997; 38:797-802

35. Laissy JP, Normand G, Monroc M, et al. Spontaneous intracerebral hematomas from vascular causes: predictive value of CT compared with angiography. Neuroradiology 1991;33:291-95

36. Douglas MA, Haerer AF. Long-term prognosis of hypertensive intracerebral hemorrhage. Stroke 1982;13:488-91

37. Fisher CM. Pathological observations in hypertensive cerebral hemorrhage. J Neuropathol Exp Neurol 1971;30:536-50

38. Russell DS. Spontaneous intracranial haemorrhage. Proc R Soc Med 1954;47:689-93

39. Toffol GJ, Biller J, Adams HP Jr. Nontraumatic intracerebral hemorrhage in young adults. Arch Neurol 1987;44:483-85

40. Hino A, Fujimoto M, Yamaki T, et al. Value of repeat angiography in patients with spontaneous subcortical hemorrhage. Stroke 1998;29: 2517-21

41. McCormick WF, Rosenfield DB. Massive brain hemorrhage: a review of 144 cases and an examination of their causes. Stroke 1973;4: $946-54$

42. Steiner T, Diringer MN, Schneider D, et al. Dynamics of intraventricular hemorrhage in patients with spontaneous intracerebral hemorrhage: risk factors, clinical impact, and effect of hemostatic therapy with recombinant activated factor VII. Neurosurgery 2006; 59:767-73, discussion 773-74

43. Thompson AL, Kosior JC, Gladstone DJ, et al. Defining the CT angiography 'spot sign' in primary intracerebral hemorrhage. Can J Neurol Sci 2009;36:456-61

44. Wang YH, Fan JY, Luo GD, et al. Hematoma volume affects the accuracy of computed tomographic angiography 'spot sign' in predicting hematoma expansion after acute intracerebral hemorrhage. Eur Neurol 2011;65:150-55

45. Demchuk A, Tymchuck S, O'Reilly C, et al. Multicentre prospective study demonstrates feasibility of CT-angiography in intracerebral hemorrhage and validity of "spot sign" for hematoma expansion prediction (PREDICT/Sunnybrook ICH CTA Study Group). In: Proceedings of the International Stroke Conference, New Orleans, Louisiana. February 20-22, 2008

46. Delgado Almandoz JE, Yoo AJ, Stone MJ, et al. Systematic characterization of the computed tomography angiography spot sign in primary intracerebral hemorrhage identifies patients at highest risk for hematoma expansion: the spot sign score. Stroke 2009;40:29943000

47. Becker K, Tirschwell D. Stroke: 'spotting' patients at the highest risk of hematoma growth. Nat Rev Neurol 2009;5:526-28
48. Delgado Almandoz JE, Yoo AJ, Stone MJ, et al. Extended analysis of the spot sign score's performance. Nat Rev Neurol 2010;6:152

49. Aviv RI, Gladstone D, Goldstein J, et al. Contrast extravasation predicts hematoma growth: where to now? AJNR Am J Neuroradiol 2008;29:E80

50. Evans A, Demchuk A, Symons SP, et al. The spot sign is more common in the absence of multiple prior microbleeds. Stroke 2010;41: 2210-17

51. Gazzola S, Aviv RI, Gladstone DJ, et al. Vascular and nonvascular mimics of the CT angiography "spot sign" in patients with secondary intracerebral hemorrhage. Stroke 2008;39:1177-83

52. Willinsky RA, Taylor SM, TerBrugge K, et al. Neurologic complications of cerebral angiography: prospective analysis of 2,899 procedures and review of the literature. Radiology 2003;227:522-28

53. Menke J, Larsen J, Kallenberg K. Diagnosing cerebral aneurysms by computed tomographic angiography: meta-analysis. Ann Neurol 2011;69:646-54

54. Wong GK, Siu DY, Abrigo JM, et al. Computed tomographic angiography and venography for young or nonhypertensive patients with acute spontaneous intracerebral hemorrhage. Stroke 2011;42: 211-13

55. Chappell ET, Moure FC, Good MC. Comparison of computed tomographic angiography with digital subtraction angiography in the diagnosis of cerebral aneurysms: a meta-analysis. Neurosurgery 2003;52:624-31, discussion 630-31

56. Mehran R, Nikolsky E. Contrast-induced nephropathy: definition, epidemiology, and patients at risk. Kidney Int Suppl 2006:S11-15

57. Rudnick MR, Goldfarb S, Wexler L, et al. Nephrotoxicity of ionic and nonionic contrast media in 1196 patients: a randomized trial. The Iohexol Cooperative Study. Kidney Int 1995;47:254-61

58. Krol AL, Dzialowski I, Roy J, et al. Incidence of radiocontrast nephropathy in patients undergoing acute stroke computed tomography angiography. Stroke 2007;38:2364-66

59. Aulicky P, Mikulik R, Goldemund D, et al. Safety of performing CT angiography in stroke patients treated with intravenous thrombolysis. J Neurol Neurosurg Psychiatry 2010;81:783-87

60. Cramer BC, Parfrey PS, Hutchinson TA, et al. Renal function following infusion of radiologic contrast material: a prospective controlled study. Arch Intern Med 1985;145:87-89

61. Heller CA, Knapp J, Halliday J, et al. Failure to demonstrate contrast nephrotoxicity. Med J Aust 1991;155:329-32

62. Benko A, Fraser-Hill M, Magner P, et al. Canadian Association of Radiologists: consensus guidelines for the prevention of contrastinduced nephropathy. Can Assoc Radiol J 2007;58:79-87

63. Rossaint R, Bouillon B, Cerny V, et al. Management of bleeding following major trauma: an updated European guideline. Crit Care 2010;14:R52

64. Spahn DR, Cerny V, Coats TJ, et al. Management of bleeding following major trauma: a European guideline. Crit Care 2007; 11:R17

65. Biffl WL, Cothren CC, Moore EE, et al. Western Trauma Association critical decisions in trauma: screening for and treatment of blunt cerebrovascular injuries. J Trauma 2009;67:1150-53

66. Eastman AL, Muraliraj V, Sperry JL, et al. CTA-based screening reduces time to diagnosis and stroke rate in blunt cervical vascular injury. J Trauma 2009;67:551-56

67. Caro JJ, Trindade E, McGregor M. The risks of death and of severe nonfatal reactions with high- vs low-osmolality contrast media: a meta-analysis. AJR AM J Roentgenol 1991;156:825-32

68. Cohnen M, Wittsack HJ, Assadi S, et al. Radiation exposure of patients in comprehensive computed tomography of the head in acute stroke. AJNR Am J Neuroradiol 2006;27:1741-45

69. Brenner DJ, Hall EJ. Computed tomography-an increasing source of radiation exposure. $N$ Engl J Med 2007;357:2277-84

70. Sodickson A, Baeyens PF, Andriole KP, et al. Recurrent CT, cumulative radiation exposure, and associated radiation-induced cancer risks from CT of adults. Radiology 2009;251:175-84 